\title{
Distributed Optimal Day-Ahead Scheduling in a Smart Grid: A Trade-Off among Consumers, Power Suppliers, and Transmission Owners
}

\author{
Xiong $\mathrm{Hu}^{1}$ and Zhi-Wei Liu ${ }^{2}$ \\ ${ }^{1}$ Department of Automation, School of Power and Mechanical Engineering, Wuhan University, Wuhan 430072, China \\ ${ }^{2}$ School of Automation, Huazhong University of Science and Technology, Wuhan 430074, China \\ Correspondence should be addressed to Zhi-Wei Liu; zwliu@sina.com
}

Received 19 June 2017; Accepted 14 November 2017; Published 19 December 2017

Academic Editor: Roberto Natella

Copyright (C) 2017 Xiong Hu and Zhi-Wei Liu. This is an open access article distributed under the Creative Commons Attribution License, which permits unrestricted use, distribution, and reproduction in any medium, provided the original work is properly cited.

\begin{abstract}
To cope with the challenges due to increasing peak load, an optimal day-ahead scheduling problem for social welfare maximization is proposed, in which not only the comfort level of consumers and costs of power suppliers but also the power losses in transmission and operation costs of transmission owners are taken into account. Then this optimal day-ahead scheduling problem is reformulated and solved via the alternating direction method of multipliers (ADMM), by which fast convergence is guaranteed and the privacy of participants is ensured, in a distributed manner. Specifically, in the proposed distributed optimal day-ahead scheduling, the hourly prices for consumers are divided into hourly supply prices and hourly delivery prices, which will be updated by the independent system operator based on the hourly demand-supply situations and hourly demand-delivery situations, respectively. And the consumers, power suppliers, and transmission owners make their individual optimal day-ahead scheduling based on their individual hourly prices, hourly supply prices, and hourly delivery prices, respectively, until the hourly demand-supply balances and hourly demand-delivery balances are achieved. Effectiveness of the proposed distributed optimal day-ahead scheduling is verified by the cases studied.
\end{abstract}

\section{Introduction}

Recently, increasing peak load due to economic development and replacing fossil fuels with electricity power has resulted in serious challenges to the power grid, such as increasing the operation costs and decreasing the reliability of power grid $[1,2]$. To cope with these challenges, day-ahead scheduling, which is widely recognized as an indirectly and effective approach for costs reducing and peak load shaving, has been intensively discussed [3-6].

So far, there is plenty of literature about the day-ahead scheduling, minimizing costs of power suppliers [7, 8], minimizing energy costs of consumers [9], minimizing costs of utility company and payments of consumers [10], or minimizing the day-ahead operation costs of integrated urban energy system [11]. From the social perspective, it is desired to maximize the sum of comfort level of consumers and meanwhile minimize the costs of utility companies. This is also called the social welfare maximization and has attracted much attention [12-16]. Besides, distributed coordination approaches $[17,18]$, which are widely applied in many other fields, have been introduced into the day-ahead scheduling in a smart grid recently.

As well known, power system consists of not only power generation and consumption but also power transmission, and the costs of transmission owners, which includes power losses in transmission and operation costs, play important part in the social welfare. Besides, since improper dayahead scheduling might challenge the operation of power transmission and cause congestions, the capacity limits of tie lines should be considered in the day-ahead scheduling, which makes the day-ahead scheduling more complex but more reasonable.

While most of the mentioned literature $[10,12,16]$ assumes that all the consumers and generators are connected to the same bus, few literatures [13] have considered the 
capacity limits of tie lines, but the costs of transmission owners are ignored.

Besides, although many distributed theories $[19,20]$ and decomposition based approaches have been proposed to solve the day-ahead scheduling in distributed manners, the convergence rates of some approaches, such as subgradient projection method, are not fast enough, and they are highly dependent on the choice of step size [16]. Moreover, for most decomposition based approaches, in certain cases, their convergence criteria may not hold and modified decomposition method is required [21].

Nowadays, low cost communications technologies enable more cost-reflective price for electricity services, which can finally animate the day-ahead scheduling and facilitate the optimization of social welfare. Then, in this paper, a distributed optimal day-ahead scheduling for social welfare maximization is proposed, in which not only the comfort level of consumers and costs of power suppliers but also the costs of transmission owners are considered.

In the proposed distributed optimal day-ahead scheduling, the participants are consumers, power suppliers, and transmission owners. The day-ahead hourly prices for consumers are divided into hourly supply prices and hourly delivery prices, and each consumer has signed contracts with a power supplier and several transmission owners who govern the tie lines connecting himself to the power supplier chosen by him. Since each power supplier has his individual hourly supply prices which indicate his individual hourly demand-supply situations and each transmission owner has his individual hourly delivery prices which indicate his individual hourly demand-delivery situations, then each consumer has his individual hourly prices.

In the beginning, consumers, power suppliers, and transmission owners submit their individual initial day-ahead schedules to the independent system operator (ISO). Most of the time, there are hourly demand-supply differences for each power supplier and hourly demand-delivery differences for each transmission owner. Then based on experience and their individual hourly differences, ISO proposes hourly supply prices for each power supplier and hourly delivery prices for each transmission owner and broadcasts the hourly demand-supply differences, hourly demand-delivery differences, hourly prices, hourly supply prices, and hourly delivery prices to the corresponding participants.

First, based on the related hourly demand-supply differences, hourly demand-delivery differences, and his individual hourly prices, each consumer makes his optimal day-ahead hourly demands and submits them to the ISO. With the updated day-ahead hourly demands of consumers, the hourly demand-supply differences and hourly demand-delivery differences are half updated by the ISO and broadcast to the corresponding power suppliers and transmission owners, respectively.

Next, each power supplier makes optimal day-ahead hourly supplies based on his individual hourly supply prices and half updated hourly demand-supply differences and submits them to the ISO as well. Similarly, each transmission owner makes optimal day-ahead hourly deliveries based on his individual hourly delivery prices and half updated hourly demand-delivery differences and submits them to the ISO. Then with the updated day-ahead schedules of all the participants, all of the hourly demand-supply differences and hourly demand-delivery differences are fully updated, and all of the hourly supply prices and hourly delivery prices are updated by the ISO.

The newest hourly demand-supply differences, hourly demand-delivery differences, hourly prices, hourly supply prices, and hourly delivery prices will be broadcast to the corresponding participants, and this process will be ended until all the hourly demand-supply balances and hourly demand-delivery balances are achieved.

The contributions of this paper are summarized in the following. First, quite different from the existing literature $[10,12,13,16]$, in which only the comfort level of consumers and fuel costs of power suppliers are considered, in this paper, the power losses in transmission and operation costs of transmission owners are considered as well. Accordingly, for each transmission owner, the hourly delivery prices, which indicate his individual hourly demand-delivery situations, are introduced.

Second, the costs of power suppliers associated with hourly supplies variability, which play an important role in hourly supply prices [22], have been taken into account. That makes the proposed social welfare more comprehensive.

Third, with the aid of the ISO, the proposed distributed optimal day-ahead scheduling can be properly matched with the framework of alternating direction method of multipliers (ADMM), by which a global and fast convergence is guaranteed and the privacy of participants is ensured.

This paper is organized as follows. The proposed distributed optimal day-ahead scheduling problem is formulated in Section 2. The design of the proposed day-ahead scheduling and the corresponding algorithms are presented in Section 3. The cases studied are illustrated in Section 4, and the last section is the conclusion.

\section{Problem Formulation}

In this section, topology of the smart grid is illustrated in Figure 1, and a highly coupled day-ahead scheduling problem for social welfare maximization is proposed, in which not only the comfort level of consumers and costs of power suppliers but also the power losses in transmission and operation costs of transmission owners are considered.

2.1. Cost Function of Transmission Owner. In this paper, assume that the voltage in the power grid is constant; denote the set of tie lines by $L$; as well known, for each tie line $l$, power losses in transmission can be written as

$$
P_{\text {loss }, l}=\frac{P_{l}^{2}+Q_{l}^{2}}{U_{n}^{2}} R_{l}, \quad \forall l \in L,
$$

where $P_{l}$ is the active power, $Q_{l}$ is the reactive power, $U_{n}$ is the nominal voltage, $R_{l}$ is the equivalent resistance of tie line l. Denoting the line power factor of tie line $l$ by $\cos \varphi$, we have

$$
\cos ^{2} \varphi=\frac{P_{l}^{2}}{P_{l}^{2}+Q_{l}^{2}}, \quad \forall l \in L .
$$




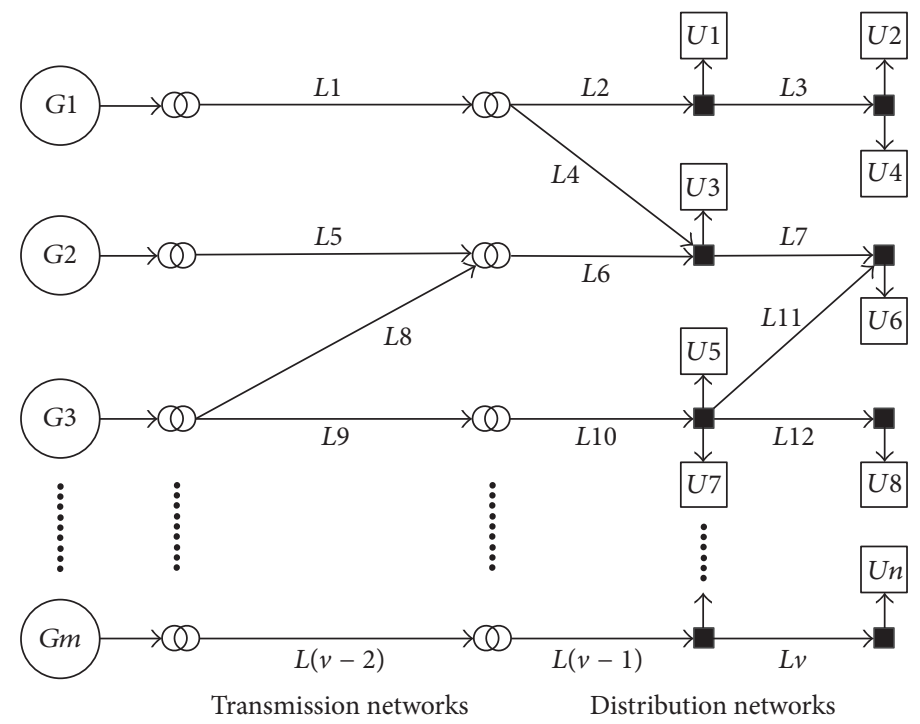

FIgURE 1: Topology of the smart grid. Note that $U_{n}$ is the $n$th consumers aggregations.

Then from (1), define $a_{l}=2 R_{l} /\left(U_{n} \cos \varphi\right)^{2}$ and denote the set of time slots by $T$, we formulate the cost function of tie line $l$ in time slot $j$ as

$$
f_{l, j}\left(P_{l, j}\right)=\frac{a_{l}}{2} P_{l, j}^{2}+b_{l} P_{l, j}+c_{l}, \quad \forall l \in L, \forall j \in T,
$$

where $P_{l, j}$ is the active power delivered by tie line $l$ in time slot $j, b_{l}$ and $c_{l}$ are the parameters related to operation costs of transmission owner $l$.

Define $\mathscr{L}_{l}=\left(P_{l, 1}, \ldots, P_{l, t}\right)^{T} ; t$ is the number of time slots; then the cost function of tie line $l$ can be formulated as

$$
F_{l}\left(\mathscr{L}_{l}\right)=\sum_{j \in T} f_{l, j}\left(P_{l, j}\right), \quad \forall l \in L .
$$

2.2. Cost Function of Power Supplier. For the fuel costs of power suppliers, we choose the most commonly used quadratic function. Denote the set of suppliers by $G, \mathscr{G}_{g}=$ $\left(P_{g, 1}, \ldots, P_{g, t}\right)^{T}$; then, for supplier $g$, his fuel costs in the next day $f_{g 1}$ can be formulated as

$$
f_{g 1}\left(\mathscr{G}_{g}\right)=\sum_{j \in T}\left(\frac{a_{g}}{2} P_{g, j}^{2}+b_{g} P_{g, j}+c_{g}\right), \quad \forall g \in G,
$$

where $P_{g, j}$ is the active power generated by power supplier $g$ in time slot $j ; a_{g}, b_{g}$, and $c_{g}$ are positive parameters.

Besides, for supplier $g$, the costs associated with hourly supplies variability are formulated as the following quadratic function:

$$
f_{g 2}\left(\mathscr{G}_{g}\right)=\frac{\eta_{g}}{2} \sum_{j=1}^{t-1}\left(P_{g, j}-P_{g, j+1}\right)^{2}, \quad \forall g \in G
$$

Hence the costs function of power suppliers $g$ can be formulated as

$$
F_{g}\left(\mathscr{G}_{g}\right)=f_{g 1}\left(\mathscr{G}_{g}\right)+f_{g 2}\left(\mathscr{G}_{g}\right), \quad \forall g \in G .
$$

2.3. Utility Function. Denote the set of consumers by $U$, for each consumer $i$ in each time slot $j$, the utility function $u_{i j}$, which values his comfort level, can be formulated as follows [12]:

$$
u_{i, j}\left(x_{i, j}, \omega_{i, j}\right)= \begin{cases}\omega_{i, j} x_{i, j}-\frac{\alpha}{2} x_{i, j}^{2}, & 0 \leq x_{i, j} \leq \frac{\omega_{i, j}}{\alpha}, \\ \frac{\omega_{i, j}^{2}}{2 \alpha}, & x_{i, j} \geq \frac{\omega_{i, j}}{\alpha},\end{cases}
$$

where $x_{i, j}$ is the aggregate demand of consumer $i$ in time slot $j, \omega_{i, j}$ is the parameter related to comfort level of consumer $i$ in time slot $j$, a higher $\omega_{i, j}$ implies a higher utility value, and $\alpha$ is a predefined parameter related to the electricity costs of consumer.

In essence, with the assumption that power suppliers have quadratic costs functions, for consumer $i$ and the power supplier $g$ chosen by consumer $i, \alpha$ is dependent on the active power $P_{g, j}$ generated by power supplier $g$ in time slot $j$. Generally, consumer $i$ has a small $\alpha$ for off-peak time slots and a large $\alpha$ for on-peak time slots, $i \in U, g \in G, j \in T$. In this paper, we can assign different $\alpha$ to different time slots for each consumer $i$ based on general daily demand curve of consumers, such as $0.02,0.3$, and 0.5 for off-peak, mid-peak, and on-peak time slots, respectively [12]. But for simplicity, we assign a middle $\alpha$ for all the consumers in all the time slots, and this has no substantial impact on the proposed distributed day-ahead scheduling.

Define $\mathscr{X}_{i}=\left(x_{i, 1}, \ldots, x_{i, t}\right)^{T}$; then the utility function of consumer $i$ can be formulated as

$$
F_{i}\left(\mathscr{X}_{i}\right)=\sum_{j \in T} u_{i, j}\left(x_{i, j}\right), \quad \forall i \in U .
$$

2.4. Proposed Optimal Day-Ahead Scheduling Problem. Define $\mathbf{x}=\left(\mathscr{X}_{1}{ }^{T}, \ldots, \mathscr{X}_{n}{ }^{T}\right)^{T}, n$ is the number of consumers, $\mathbf{Z}_{1}=\left(\mathscr{G}_{1}^{T}, \ldots, \mathscr{G}_{m}{ }^{T}\right)^{T}, m$ is the number of power suppliers, 
$\mathbf{Z}_{2}=\left(\mathscr{L}_{1}{ }^{T}, \ldots, \mathscr{L}_{v}{ }^{T}\right)^{T}$, and $v$ is the number of tie lines. Then the objective function for social welfare maximization is formulated as

$$
\begin{aligned}
W\left(\mathbf{x}, \mathbf{Z}_{1}, \mathbf{Z}_{2}\right)= & \sum_{i \in U} F_{i}\left(\mathscr{X}_{i}\right)-\sum_{g \in G} F_{g}\left(\mathscr{G}_{g}\right) \\
& -\sum_{l \in L} F_{l}\left(\mathscr{L}_{l}\right) .
\end{aligned}
$$

For day-ahead scheduling in this paper, we assign load curtailment to real-time market [23] and concentrate on load shifting. That means the total demand for each consumer $i$ in the next day is a constant in this scheduling and we have

$$
\sum_{j \in T} x_{i j}=D_{i}, \quad \forall i \in U
$$

where $D_{i}$ is the total demand for consumer $i$ in the next day. And for each $x_{i, j}, i \in U, j \in T$, we have

$$
\underline{x}_{i, j} \leq x_{i, j} \leq \bar{x}_{i, j}, \quad \forall i \in U, \forall j \in T \text {, }
$$

where $\underline{x}_{i, j}$ and $\bar{x}_{i, j}$ are the lower and upper bounds for demand of consumer $i$ in time slot $j$, respectively.

For each power supplier $g$ in time slot $j$, its output $P_{g, j}$ should equal the sum of demands of consumers who purchase power from him. In this paper, we assume that the consumers in the same aggregation choose the same power supplier and denote the set of consumers who purchase power from power supplier $g$ by $U_{g}$; then we have

$$
\sum_{i \in U_{g}} x_{i j}=P_{g, j}, \quad \forall g \in G, \forall j \in T,
$$

and we have

$$
\underline{P}_{g, j} \leq P_{g, j} \leq \bar{P}_{g, j}, \quad \forall g \in G, \forall j \in T,
$$

where $\underline{P}_{g, j}$ and $\bar{P}_{g, j}$ are the lower and upper bounds for $P_{g, j}$, respectively.

Besides, for each transmission owner $l$, his active power delivery should equal the sum of demands of consumers who have signed a transmission contract with him. Similarly, we assume that the consumers in the same aggregation choose the same transmission owners and denote the set of consumers who have signed a transmission contract with transmission owner $l$ by $U_{l}$; then we have

$$
\sum_{i \in U_{l}} x_{i j}=P_{l, j}, \quad \forall l \in L, \forall j \in T,
$$

and we have

$$
\underline{P}_{l, j} \leq P_{l, j} \leq \bar{P}_{l, j}, \quad \forall l \in L, \forall j \in T,
$$

where $\underline{P}_{l, j}$ and $\bar{P}_{l, j}$ are the lower and upper bounds for $P_{l, j}$, respectively.
Therefore, the objective function for social welfare maximization can be formulated as follows:

$$
\begin{array}{ll}
\min & F\left(\mathbf{x}, \mathbf{Z}_{1}, \mathbf{Z}_{2}\right) \\
& =\sum_{g \in G} F_{g}\left(\mathscr{G}_{g}\right)+\sum_{l \in L} F_{l}\left(\mathscr{L}_{l}\right)-\sum_{i \in U} F_{i}\left(\mathscr{X}_{i}\right), \\
\text { s.t. } & (11),(12),(13),(14),(15),(16) .
\end{array}
$$

where $D_{i}$ are the inputs variables, $i \in U$, and $\mathbf{x}, \mathbf{Z}_{1}, \mathbf{Z}_{2}$ are the decision variables. According to (6), (11), (13), and (15), it can be seen that the proposed day-ahead scheduling problem (17) is highly coupled.

\section{Distributed Optimal Day-Ahead Scheduling}

To achieve a distributed and fast optimal day-ahead scheduling in this paper, the proposed optimal day-ahead scheduling problem is reformulated at first; then it is solved via the $\mathrm{ADMM}$ in a distributed manner.

3.1. An Overview on ADMM. As well known, a standard form of ADMM, the details of which can be found in [24], solves the following problem:

$$
\begin{aligned}
\min & G_{1}(\mathbf{x})+G_{2}(\mathbf{z}), \\
\text { w.r.t. } & \mathbf{x} \in \mathscr{C}_{1}, \\
& \mathbf{z} \in \mathscr{C}_{2}, \\
\text { s.t. } & \mathbf{A x}=\mathbf{z} .
\end{aligned}
$$

Assume $\mathbf{z} \in \mathscr{R}^{m}$, assign a Lagrange multiplier vector $\boldsymbol{\rho} \in \mathscr{R}^{m}$ to the equality constraint $\mathbf{A x}=\mathbf{z}$, and we get the following augmented Lagrange function:

$$
\begin{aligned}
L(\mathbf{x}, \mathbf{z}, \boldsymbol{\rho})= & G_{1}(\mathbf{x})+G_{2}(\mathbf{z})+\boldsymbol{\rho}^{T}(\mathbf{A} \mathbf{x}-\mathbf{z}) \\
& +\frac{c}{2}\|\mathbf{A} \mathbf{x}-\mathbf{z}\|_{2}^{2},
\end{aligned}
$$

where $c$ is an arbitrary positive constant. Then the search for a constraint saddle point of the augmented Lagrange function is performed with an alternating procedure that starts from arbitrary initials $\mathbf{z}(0)$ and $\mathbf{p}(0)$ and iteratively updates entries as follows:

$$
\begin{aligned}
& \mathbf{x}(k+1) \\
& =\arg \min _{\mathbf{x} \in \mathscr{C}_{1}}\left\{G_{1}(\mathbf{x})+\boldsymbol{\rho}^{T} \mathbf{A} \mathbf{x}+\frac{c}{2}\|\mathbf{A} \mathbf{x}-\mathbf{z}(k)\|_{2}^{2}\right\}, \\
& \mathbf{z}(k+1) \\
& \quad=\arg \min _{\mathbf{z} \in \mathscr{C}_{2}}\left\{G_{2}(\mathbf{z})-\boldsymbol{\rho}^{T} \mathbf{z}+\frac{c}{2}\|\mathbf{A x}(k+1)-\mathbf{z}\|_{2}^{2}\right\}, \\
& \boldsymbol{\rho}(k+1)=\boldsymbol{\rho}(k)+c(\mathbf{A x}(k+1)-\mathbf{z}(k+1)),
\end{aligned}
$$

When $G_{1}$ and $G_{2}$ are convex functions, and $\mathscr{C}_{1}$ is a compact set or else the matrix $\mathbf{A}^{T} \mathbf{A}$ is invertible, ADMM converges to a unique stable point, which is assured to be a constraint saddle point for $L$, hence the optimal solution. 
3.2. Design of Distributed Optimal Day-Ahead Scheduling. First, according to problem (18) solved by ADMM, we reformulate the proposed optimal day-ahead scheduling problem (17) as follows.

Function $G_{1}(\mathbf{x})$ of the proposed day-ahead scheduling is formulated as

$$
G_{1}(\mathbf{x})=-F_{1}\left(X_{1}\right)-F_{2}\left(X_{2}\right)-\cdots-F_{n}\left(X_{n}\right),
$$

which is a strictly convex function.

For each consumer $i$, his local constraints are collected in the following compact set, $i \in U$,

$$
\mathcal{Q}_{i}=\left\{\mathscr{X}_{i} \mid \sum_{j \in T} x_{i j}=D_{i}, \underline{x}_{i, j} \leq x_{i, j} \leq \bar{x}_{i, j}, \forall j \in T\right\}
$$

and then the set $\mathscr{C}_{1}$ can be denoted by the Cartesian product

$$
\mathscr{C}_{1}=\mathbb{Q}_{1} \times \mathbb{Q}_{2} \times \cdots \times \mathbb{Q}_{n}
$$

which is a compact set.

Let $\left(\mathbf{Z}_{1}^{T}, \mathbf{Z}_{2}^{T}\right)^{T}=\mathbf{z}$; then function $G_{2}(\mathbf{z})$ for the proposed day-ahead scheduling is formulated as

$$
G_{2}(\mathbf{z})=\sum_{g \in G} F_{g}\left(\mathscr{G}_{g}\right)+\sum_{l \in L} F_{l}\left(\mathscr{L}_{l}\right)
$$

which is a strictly convex function as well.

For each power supplier $g$, his local constraints are collected in the following set, $g \in G$ :

$$
\mathcal{S}_{g}=\left\{\mathscr{G}_{g} \mid \underline{P}_{g, j} \leq P_{g, j} \leq \bar{P}_{g, j}, \forall j \in T\right\},
$$

and, for each transmission owner $l$, his local constraints are collected in the following set, $l \in L$ :

$$
\mathscr{R}_{l}=\left\{\mathscr{L}_{l} \mid \underline{P}_{l, j} \leq P_{l, j} \leq \bar{P}_{l, j}, \forall j \in T\right\},
$$

and then the set $\mathscr{C}_{2}$ can be denoted by the Cartesian product:

$$
\mathscr{C}_{2}=\mathcal{S}_{1} \times \mathcal{S}_{2} \times \cdots \times \mathcal{S}_{m} \times \mathscr{R}_{1} \times \mathscr{R}_{2} \times \cdots \times \mathscr{R}_{v} .
$$

The equation constraints $\mathbf{A x}=\mathbf{z}$ can be deduced directly from (13) and (15), where $\mathbf{A} \in \mathscr{R}^{(m+v) t \times n t}$. Define $\boldsymbol{\varrho}_{g}=\left(\rho_{g, 1}, \ldots, \rho_{g, t}\right)^{T}, g \in G, \boldsymbol{\rho}_{G}=\left(\boldsymbol{\varrho}_{1}^{T}, \ldots, \boldsymbol{\varrho}_{m}^{T}\right)^{T}, \boldsymbol{\sigma}_{l}=$ $\left(\rho_{l, 1}, \ldots, \rho_{l, t}\right)^{T}, l \in L, \boldsymbol{\rho}_{L}=\left(\boldsymbol{\sigma}_{1}^{T}, \ldots, \boldsymbol{\sigma}_{v}^{T}\right)^{T}$, then according to the above, we have $\left(\boldsymbol{\rho}_{G}^{T}, \boldsymbol{\rho}_{L}^{T}\right)^{T}=\boldsymbol{\rho}$.

Second, the proposed optimal day-ahead scheduling problem will be solved via the ADMM in a distributed manner as illustrated in (19) and (20) as follows.

Define the $k$ th hourly demand-supply differences and $k$ th hourly demand-delivery differences, respectively, as

$$
\begin{array}{cc}
\delta_{g, j}(k)=\sum_{i \in U_{g}} x_{i, j}(k)-P_{g, j}(k) & g \in G, j \in T, \\
\delta_{l, j}(k)=\sum_{i \in U_{l}} x_{i, j}(k)-P_{l, j}(k) & l \in L, j \in T,
\end{array}
$$

where $k$ is the round number of scheduling, then we define $\pi_{g}=\left(\delta_{g, 1}, \ldots, \delta_{g, t}\right)^{T}, g \in G, \omega_{l}=\left(\delta_{l, 1}, \ldots, \delta_{l, t}\right)^{T}, l \in L$.

Assume that consumer $i_{0}$ purchases power from power supplier $g_{0}$, and denote the set of transmission owners, with whom consumer $i_{0}$ has signed transmission contracts, as $O_{i_{0}}$. Then based on (17) and (20), the objective function of each consumer $i_{0}$ can be formulated as

$$
\begin{aligned}
& \min _{\mathscr{X}_{i_{0}} \in Q_{i_{0}}}\left\{-F_{i_{0}}\left(\mathscr{X}_{i_{0}}\right)+\sum_{j \in T} \rho_{g_{0}, j}(k) x_{i_{0}, j}\right. \\
& +\sum_{l_{0} \in \mathrm{O}_{i_{0}}} \sum_{j \in T} \rho_{l_{0}, j}(k) x_{i_{0}, j} \\
& +\frac{c}{2} \sum_{j \in T}\left(x_{i_{0}, j}+\delta_{g_{0}, j}(k)-x_{i_{0}, j}(k)\right)^{2} \\
& \left.+\frac{c}{2} \sum_{l_{0} \in \mathrm{O}_{i_{0}}} \sum_{j \in T}\left(x_{i_{0}, j}+\delta_{l_{0}, j}(k)-x_{i_{0}, j}(k)\right)^{2}\right\},
\end{aligned}
$$

where $F_{i_{0}}\left(\mathscr{X}_{i_{0}}\right)$ is the comfort level of consumer $i_{0}$, Lagrange multipliers $\rho_{g_{0}, j}(k)$ and $\rho_{l_{0}, j}(k)$ represent the $k$ th supply price of power supplier $g_{0}$ and the $k$ th delivery prices of transmission owner $l_{0}$ in time slot $j$, respectively, in the $k$ th round of day-ahead scheduling, and the terms with $c$ represent the penalties set by the ISO for achieving demandsupply and demand-delivery balances.

For the consumer $i_{0}$, assume his total demand of the next day is $D_{i_{0}},\left|O_{i_{0}}\right|$ is the cardinality of the set $O_{i_{0}}, \mathscr{X}_{i_{0}}(k)=$ $\left(x_{i_{0}, 1}(k), \ldots, x_{i_{0}, j}(k), \ldots, x_{i_{0}, t}(k)\right)^{T}$. As hourly supply prices $\boldsymbol{\varrho}_{g_{0}}$, hourly demand-supply differences $\boldsymbol{\pi}_{g_{0}}$ of $g_{0}$, hourly delivery prices $\boldsymbol{\sigma}_{l_{0}}$, and hourly demand-delivery differences $\omega_{l_{0}}$ of $l_{0}, l_{0} \in O_{i_{0}}$, have been broadcast to $i_{0}$ by the ISO, define

$$
x_{i_{0}, j}(k+1)=\frac{\lambda_{i_{0}}(k)+\omega_{i_{0}, j}-\phi_{i_{0}, j}(k)-c \psi_{i_{0}, j}(k)}{\alpha+c\left(1+\left|O_{i_{0}}\right|\right)},
$$

where

$$
\begin{aligned}
\phi_{i_{0}, j}(k)= & \rho_{g_{0}, j}(k)+\sum_{l_{0} \in O_{i_{0}}} \rho_{l_{0}, j}(k), \\
\psi_{i_{0}, j}(k)= & \delta_{g_{0}, j}(k)-x_{i_{0}, j}(k) \\
& +\sum_{l_{0} \in O_{i_{0}}}\left(\delta_{l_{0}, j}(k)-x_{i_{0}, j}(k)\right),
\end{aligned}
$$

then according to subproblem (29), each consumer $i_{0}$ updates its $\mathscr{X}_{i_{0}}(k)$ in parallel as Algorithm 1 shows. The essence of Algorithm 1 is the equal incremental cost criterion; in other words, for consumer $i_{0}$, one more unit of demand in any time slot makes the same increase of his comfort level.

When consumers have submitted their updated hourly demands to the ISO, denote the half updated $(k+1)$ th hourly demand-supply differences and half updated hourly demanddelivery differences, respectively, as 


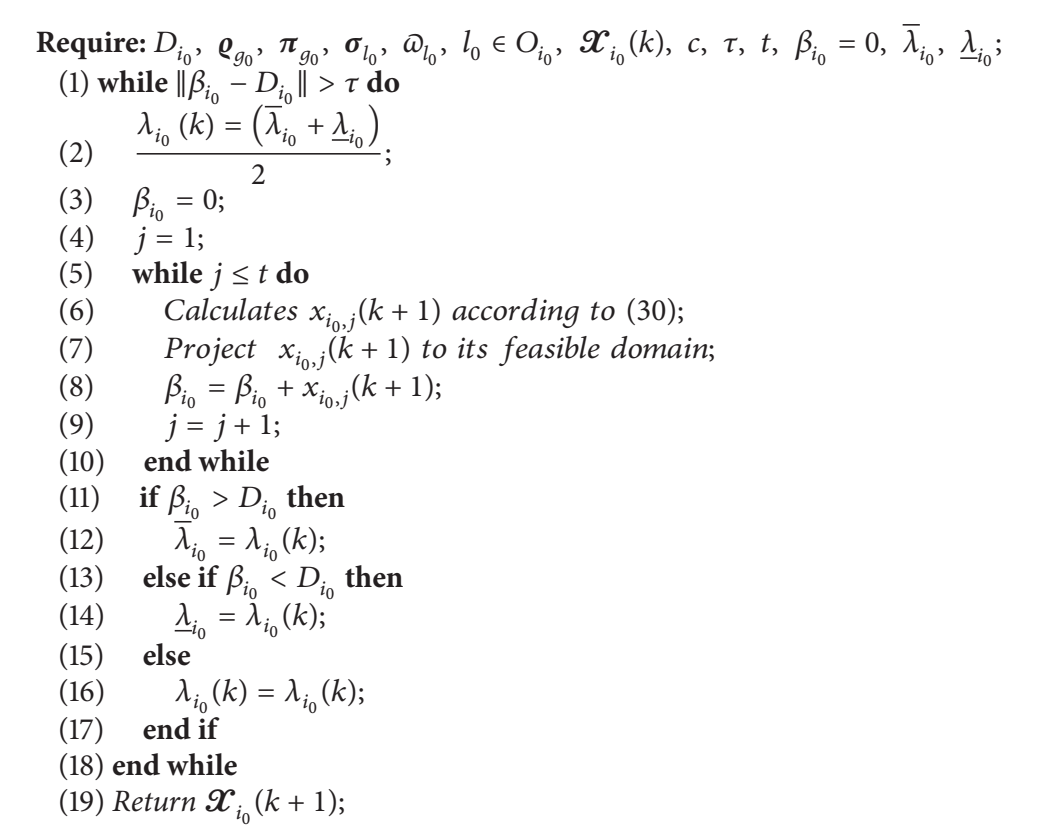

Algorithm 1: Day-ahead scheduling of consumers.

$$
\begin{array}{ll}
\widehat{\delta}_{g, j}(k+1)=\sum_{i \in U_{g}} x_{i, j}(k+1)-P_{g, j}(k) & \\
& g \in G, j \in T, \\
\widehat{\delta}_{l, j}(k+1)=\sum_{i \in U_{l}} x_{i, j}(k+1)-P_{l, j}(k) & l \in L, j \in T .
\end{array}
$$

Denote $\widehat{\pi}_{g}(k+1)=\left(\widehat{\delta}_{g, 1}(k+1), \ldots, \widehat{\delta}_{g, t}(k+1)\right)^{T}, g \in G, \widehat{\omega}_{l}(k+$ $1)=\left(\widehat{\delta}_{l, 1}(k+1), \ldots, \widehat{\delta}_{l, t}(k+1)\right)^{T}, l \in L$. Then based on the (17) and (20), the objective function of each power supplier $g_{0}, \mathfrak{F}_{g_{0}}\left(\mathscr{G}_{g_{0}}\right)$, can be formulated as

$$
\begin{gathered}
\min _{\mathscr{G}_{g_{0}} \in \mathcal{S}_{g_{0}}}\left\{\mathfrak{\mho}_{g_{0}}\left(\mathscr{G}_{g_{0}}\right)=F_{g_{0}}\left(\mathscr{G}_{g_{0}}\right)-\sum_{j \in T} \rho_{g_{0}, j}(k) P_{g_{0}, j}\right. \\
\left.+\frac{c}{2} \sum_{j \in T}\left(\widehat{\delta}_{g_{0}, j}(k+1)+P_{g_{0}, j}(k)-P_{g_{0}, j}\right)^{2}\right\},
\end{gathered}
$$

where the terms with $c$ represent the penalties set by the ISO for achieving demand-supply balance.

$$
\mathscr{G}_{g_{0}}(k)=\left(P_{g_{0}, 1}(k), \ldots, P_{g_{0}, j}(k), \ldots, P_{g_{0}, t}(k)\right)^{T} \text {; denote }
$$
$\xi_{g_{0}}(k)=\left(\xi_{g_{0}, 1}(k), \ldots, \xi_{g_{0}, j}(k), \ldots, \xi_{g_{0}, t}(k)\right)^{T}$, where

$$
\begin{aligned}
\xi_{g_{0}, j}(k) & =\left.\frac{\partial \widetilde{\mho}_{g_{0}}\left(\mathscr{G}_{g_{0}}\right)}{\partial P_{g_{0}, j}}\right|_{P_{g_{0}, j}(k)} \\
= & a_{g_{0}} P_{g_{0}, j}(k)+b_{g_{0}}-\rho_{g_{0}, j}(k)-c \widehat{\delta}_{g_{0}, j}(k+1) \\
& +\eta_{g_{0}}\left(2 P_{g_{0}, j}(k)-P_{g_{0}, j-1}(k)-P_{g_{0}, j+1}(k)\right),
\end{aligned}
$$

where $P_{g_{0}, 0}=P_{g_{0}, 1}$ and $P_{g_{0}, t+1}=P_{g_{0}, t}, g_{0} \in G, j \in T$. And define $\boldsymbol{\kappa}_{g_{0}}(k)=\left(\kappa_{g_{0}, 1}(k), \ldots, \kappa_{g_{0}, j}(k), \ldots, \kappa_{g_{0}, t}(k)\right)^{T}$, where

$$
\begin{aligned}
& \kappa_{g_{0}, j}(k)=1, \quad \underline{P}_{g_{0}, j}<P_{g_{0}, j}(k)<\bar{P}_{g_{0}, j}, \quad \xi_{g_{0}, j}(k) \neq 0, \\
& \kappa_{g_{0}, j}(k)=0, \quad \text { otherwise. }
\end{aligned}
$$

As hourly supply prices $\varrho_{g_{0}}$ and half updated hourly demandsupply differences $\widehat{\pi}_{g_{0}}(k+1)$ have been broadcast to $g_{0}$ by the ISO, then according to subproblem (33), each power supplier $g_{0}$ reschedules his hourly supplies in a distributed manner as Algorithm 2 shows. It can be seen that for power supplier $g_{0}$ the rescheduling of $\mathscr{G}_{g_{0}}(k)$ in the $k$ th round negotiation will be continued until each partial derivative of his objective function with respect to hourly output is equal to zero or that hourly output has reached its boundary.

Based on (17) and (20), the objective function of each transmission owner $l_{0}$ can be formulated as

$$
\begin{aligned}
& \min _{\mathscr{L}_{l_{0}} \in \mathscr{R}_{l_{0}}}\left\{F_{l_{0}}\left(\mathscr{L}_{l_{0}}\right)-\sum_{j \in T} \rho_{l_{0}, j}(k) P_{l_{0}, j}\right. \\
& \left.+\frac{c}{2} \sum_{j \in T}\left(\widehat{\delta}_{l_{0}, j}(k+1)+P_{l_{0}, j}(k)-P_{l_{0}, j}\right)^{2}\right\}
\end{aligned}
$$

where $F_{l_{0}}\left(\mathscr{L}_{l_{0}}\right)$ represents the power losses in transmission and operation costs; the terms with $c$ represent the penalties set by the ISO for achieving demand-delivery balance. 


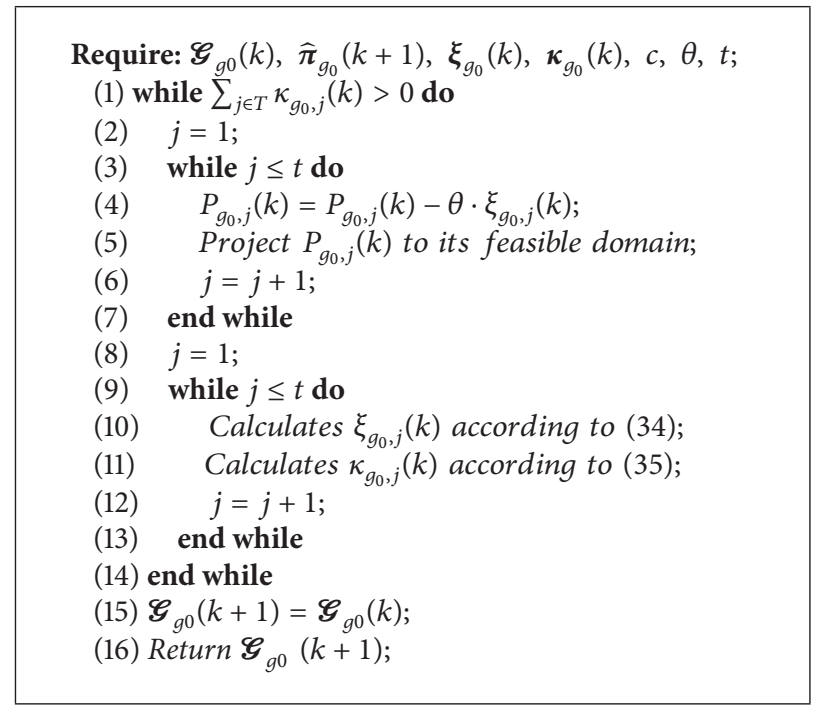

Algorithm 2: Day-ahead scheduling of power suppliers.

Define

$$
\begin{aligned}
& \widetilde{P}_{l_{0}, j}(k+1) \\
& =\frac{\rho_{l_{0}, j}(k)+c\left(\widehat{\delta}_{l_{0}, j}(k+1)+P_{l_{0}, j}(k)\right)-b_{l_{0}}}{a_{l_{0}}+c},
\end{aligned}
$$

as hourly delivery prices $\sigma_{l_{0}}$ and half updated hourly demanddelivery differences $\widehat{\widehat{\omega}}_{l_{0}}(k+1)$ have been broadcast to $l_{0}$ by the ISO; then according to subproblem (36), each transmission owner $l_{0}$ reschedules his hourly deliveries in a distributed manner as (38) shows, $l_{0} \in L, j \in T$.

$$
\begin{aligned}
& P_{l_{0}, j}(k+1) \\
& \quad= \begin{cases}\underline{P}_{l_{0}, j}, & \widetilde{P}_{l_{0}, j}(k+1)<\underline{P}_{l_{0}, j}, \\
\widetilde{P}_{l_{0}, j}(k+1), & P_{l_{0}, j} \leq \widetilde{P}_{l_{0}, j}(k+1) \leq P_{l_{0}, j}, \\
\bar{P}_{l_{0}, j}, & \widetilde{P}_{l_{0}, j}(k+1)>\bar{P}_{l_{0}, j} .\end{cases}
\end{aligned}
$$

When each power supplier $g_{0}$ and transmission owner $l_{0}$ have submitted their updated individual hourly supplies and hourly deliveries to the ISO, the $(k+1)$ th hourly demandsupply differences and hourly demand-delivery differences can be formulated, respectively, as

$$
\begin{aligned}
& \delta_{g, j}(k+1)=\sum_{i \in U_{g}} x_{i, j}(k+1)-P_{g, j}(k+1) \\
& g \in G, j \in T, \\
& \delta_{l, j}(k+1)=\sum_{i \in U_{l}} x_{i, j}(k+1)-P_{l, j}(k+1)
\end{aligned}
$$

and the hourly supply prices $\rho_{g, j}$ and hourly delivery prices $\rho_{l, j}$ will be updated as (40) shows, $g \in G, l \in L, j \in T$.

$$
\begin{gathered}
\rho_{g, j}(k+1)=\rho_{g, j}(k)+c \delta_{g, j}(k+1), \\
\rho_{l, j}(k+1)=\rho_{l, j}(k)+c \delta_{l, j}(k+1) .
\end{gathered}
$$

That is, ISO adjusts the hourly supply prices based on the hourly demand-supply differences, if demands exceed supplies, increases the supply charges, and otherwise decreases the supply charges. And the hourly delivery prices will be adjusted in the same way based on the hourly demanddelivery differences. And this scheduling process between consumers, power suppliers, and transmission owners will continue until both demand-supply and demand-delivery are balanced.

The proposed distributed optimal day-ahead scheduling can be summarized as follows.

Step 1. Consumers, suppliers, and transmission owners submit their individual initial day-ahead schedules to the ISO, and then ISO calculates the hourly differences according to (28) and set the initial hourly prices based on experience.

Step 2. ISO broadcasts the hourly prices and hourly differences to the corresponding participants.

Step 3. Consumers call Algorithm 1 and submit $\mathbf{x}(k+1)$.

Step 4. ISO broadcasts $\widehat{\boldsymbol{\delta}}(k+1)$ calculated according to (32) to the corresponding power suppliers and transmission owners.

Step 5. Suppliers and transmission owners update their individual day-ahead schedules in parallel according to Algorithm 2 and (38), respectively, and submit them to the ISO.

Step 6. ISO updates the hourly differences and hourly prices according to (39) and (40), respectively. 
TABLE 1: Contractual relationship between the consumers aggregations and power suppliers, transmission, owners.

\begin{tabular}{lcc}
\hline Consumers aggregation & Power supplier & Transmission owner \\
\hline U1 & G1 & L1, L2 \\
U2 & G1 & L1, L2, L3 \\
U3 & G2 & L2, L3, L4 \\
U4 & G2 & L5, L6 \\
U5 & G2 & L5, L6, L7 \\
\hline
\end{tabular}

TABle 2: Parameters of cost functions $\left(10^{-3}\right)$.

\begin{tabular}{cccccccccc}
\hline & G1 & G2 & L1 & L2 & L3 & L4 & L5 & L6 & L7 \\
\hline a & 1 & 0.5 & 0.22 & 0.20 & 0.26 & 0.35 & 0.25 & 0.20 & 0.23 \\
b & 100 & 100 & 20 & 18 & 21 & 23 & 19 & 19 & 22 \\
\hline
\end{tabular}

Step 7. If demand-supply and demand-delivery are balanced, this process is ended; otherwise turn to Step 2.

\section{Case Study}

In this section, we assess the convergence performance of the proposed optimal day-ahead scheduling, and some key parameters are discussed. In the cases studied, we consider a smart power grid illustrated in Figure 2, which consists of 100 consumers partitioned into 5 aggregations, 2 power suppliers, and 7 transmission owners. The contractual relationships for consumers aggregations are summarized in Table 1.

For the utility functions of consumers, the parameter $\alpha$, which has been discussed in detail in [12], is set to be 0.3 , and $c$ is chosen as 0.004 , the number of time slots is 24 , parameters $\omega_{i, j}$ are selected from 1.5 to 3 . Besides, the parameters of cost functions of power suppliers and transmission owners are presented in Table 2.

4.1. Convergence Performance and Social Welfare. The convergence performance of the proposed optimal day-ahead scheduling is presented in Figures 3 and 4. Figure 3 is the trajectories of infinity norm of hourly differences, where $\delta_{G 1}$ is the infinity norm of hourly differences of G1, and Figure 4 is the hourly supply prices trajectories of G1.

It can be seen that the infinity norm of hourly differences of all the participants and hourly supply prices of G1 achieves convergence in 10 iterations. Specifically, the proposed optimal day-ahead scheduling is carried out on a computer based on Intel(R) Core(TM) i3-4170 CPU @3.70 GHz, RAM: 8.00 GB, 64-bit Operating System, $\times 64$-based processor; the total time is 39.76 seconds. That means the proposed algorithm takes approximate 3.976 seconds per iteration. In fact, the hourly delivery prices of transmission owners, which is omitted due to limited textual paragraphs, do the same convergence performance. Then it can be concluded that the proposed distributed optimal day-ahead scheduling solved via $A D M M$ achieves convergence fast.

Figure 5 is the social welfare trajectories of the proposed distributed day-ahead scheduling. Note that the comfort level of consumers is measured in money in this paper. In essence

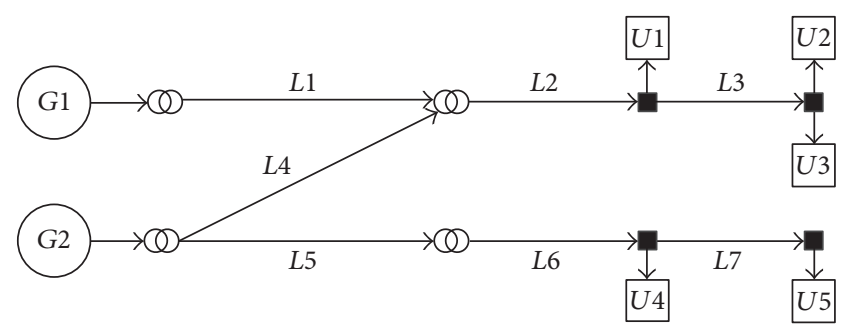

Figure 2: Topology of power grid studied.

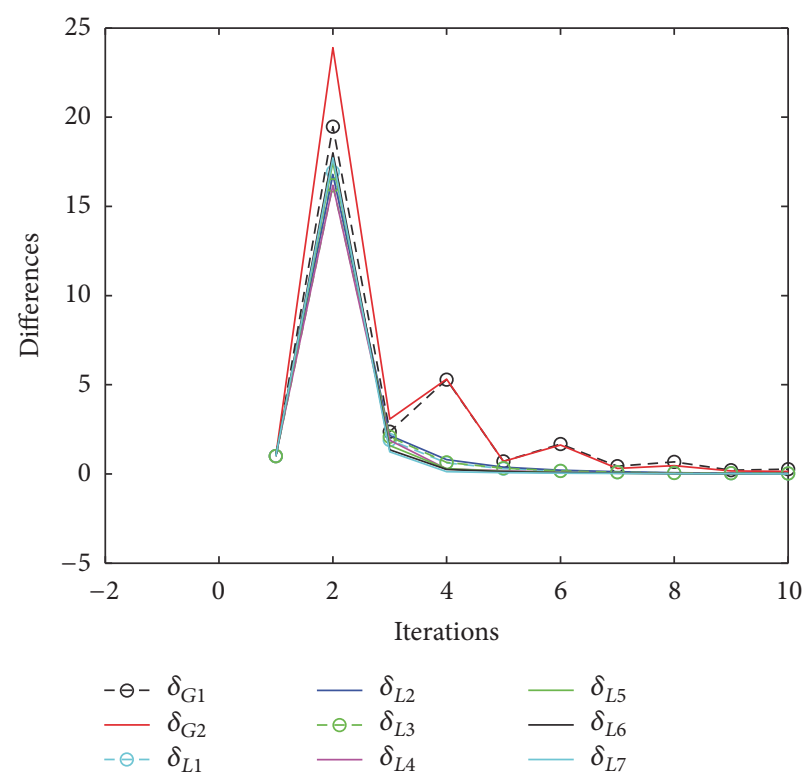

FIGURE 3: The trajectories of infinity norm of hourly differences.

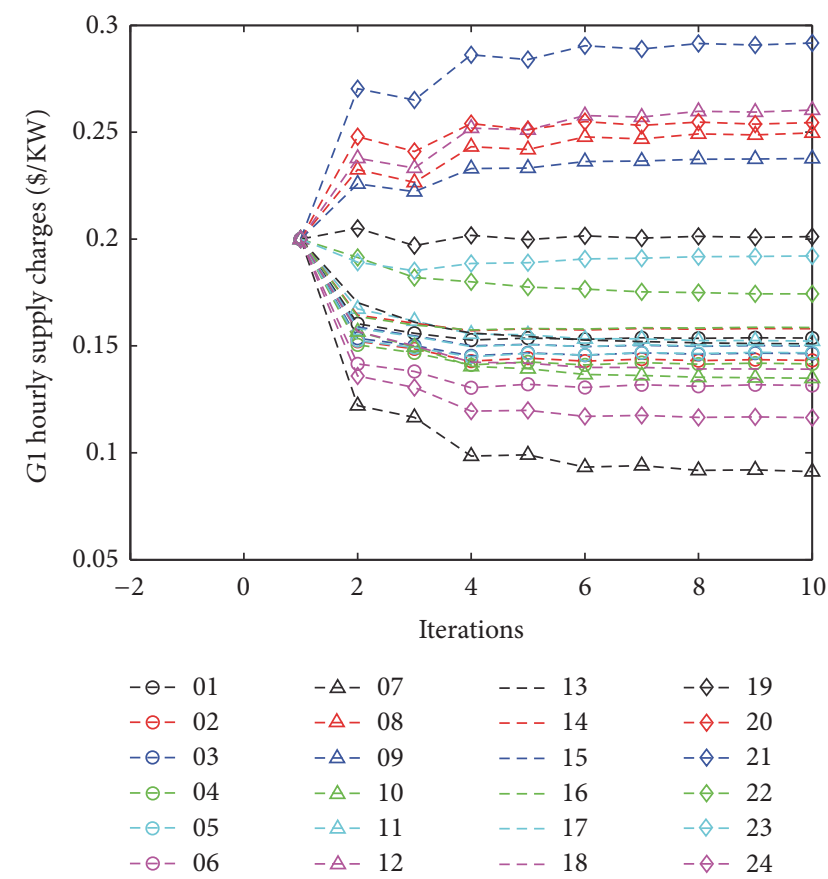

FIGURE 4: The hourly supply prices trajectories of G1. 


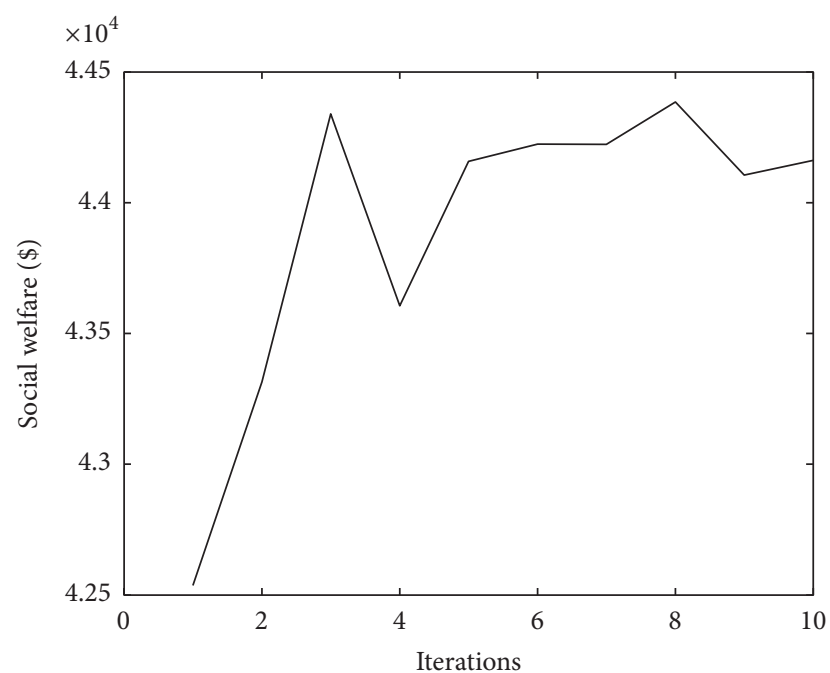

FIgURE 5: The trajectory of social welfare.

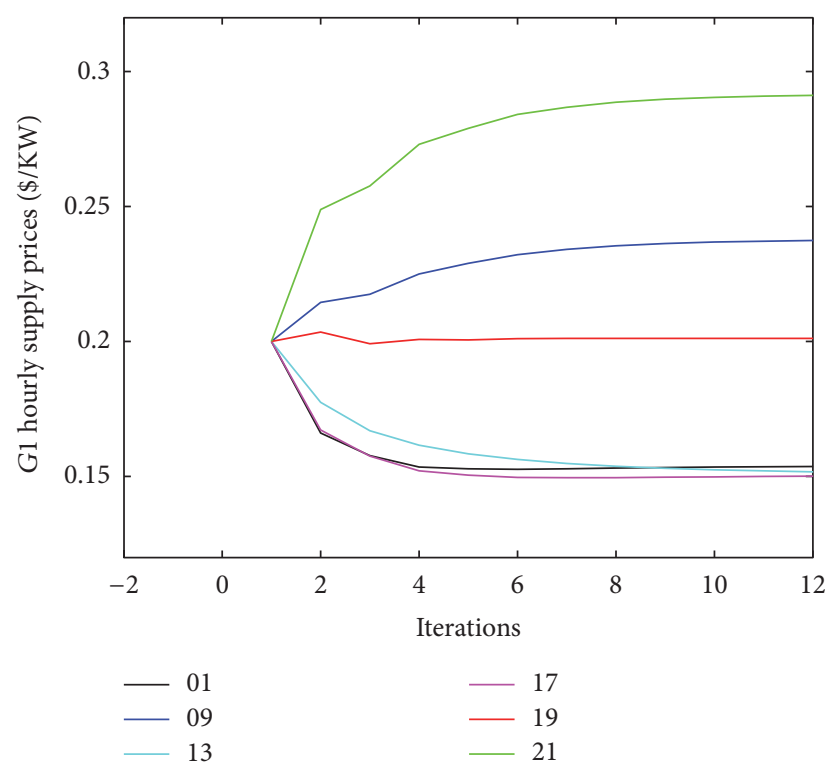

FIGURE 6: Partial hourly supply prices trajectories of G1.

the proposed social welfare is a trade-off between the comfort level of consumers and the costs of power suppliers and transmission owners. It can be seen that the social welfare is truly increased via the proposed distributed day-ahead scheduling.

4.2. Demand Shifting. Figure 6 is partial hourly supply prices trajectories of G1; Figure 7 is partial hourly delivery prices trajectories of L2; Figure 8 is the demand shifting of day-ahead scheduling of consumers aggregation U2, where "initial" and "optimal" correspond to the initial hourly demands and optimal hourly demands with $\eta=0.002$ of $\mathrm{U} 2$, respectively.

From Figure 6 and Figure 8, it can be seen that the hourly supply prices vary with the demands of consumers. With the same initial value 0.2 , the hourly supply prices increase

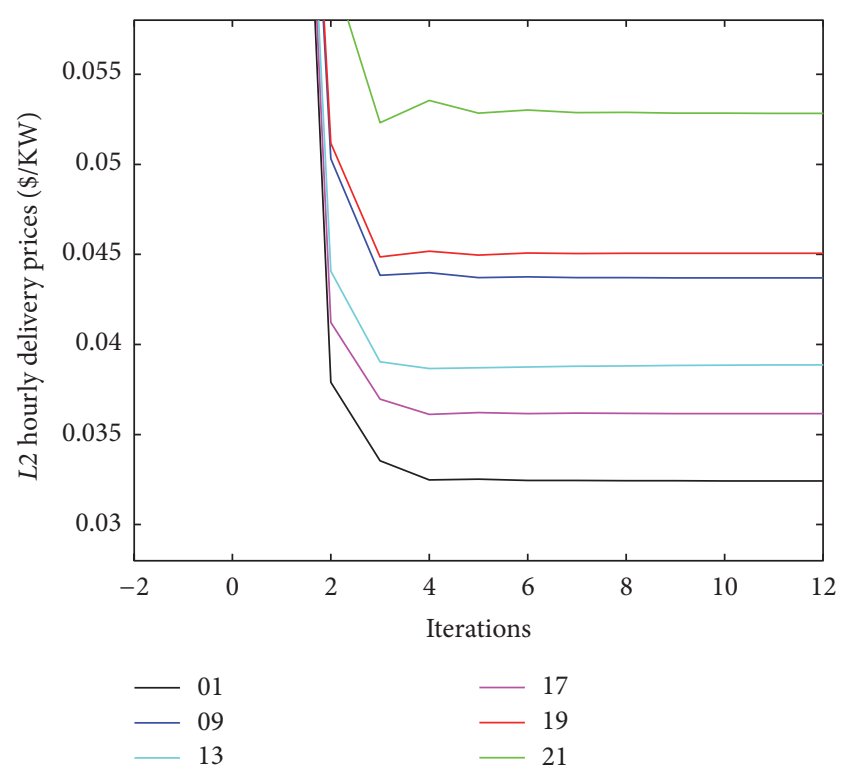

FIGURE 7: Partial hourly delivery prices trajectories of $L 2$.

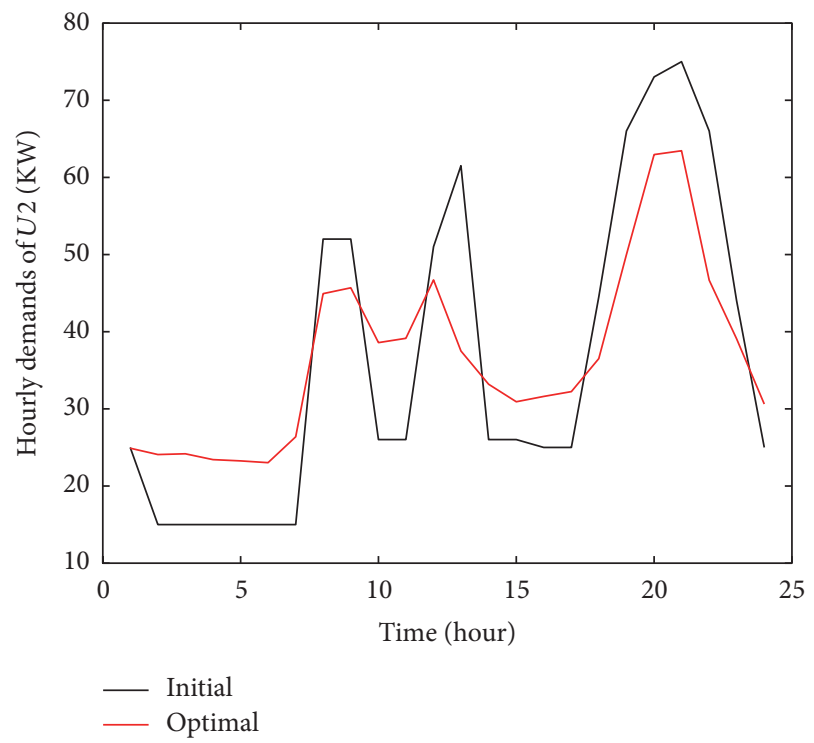

FIgURE 8: The demand shifting of day-ahead scheduling of $U 2$.

from their initial value when the demands of consumers are high and decrease from their initial value when the demands of consumers are low. From Figure 7 and Figure 8, we have the same conclusion for hourly delivery prices of transmission owners. Then accordingly, consumers will shift their demands from the hours with high electricity prices (high supply prices or high delivery prices or both) to the hours with low electricity prices, which is illustrated in Figure 8.

4.3. The Effect of $\eta$ on Day-Ahead Scheduling. Note that $\eta$ represents the costs of power suppliers associated with hourly supplies variability. The effect of $\eta$ on the proposed optimal day-ahead scheduling is illustrated in Figure 9 by the optimal 


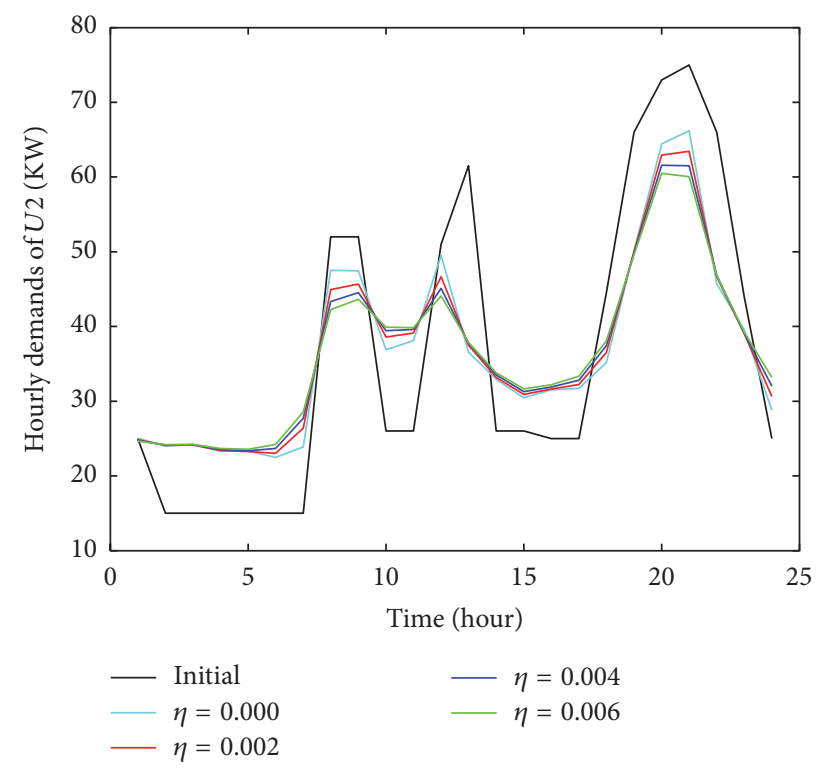

FIgURE 9: The effect of $\eta$ on optimal hourly demands of $U 2$.

day-ahead hourly demands of U2, and the optimal day-ahead scheduling of the other consumers aggregations has similar trends.

From Figure 9, it can be seen that the peak demands of U2 decrease as $\eta$ increases from 0.000 to 0.006 , that means $\eta$ plays an important role, not only in electricity price but also in reducing the peak demand of consumers. This can be explained by the response of consumers to the supply prices. As $\eta$ increases, the costs of power suppliers increase, especially the hours with dramatic supply variability which are the hours with peak demand as well, then the supply prices of these hours will be high; correspondingly consumers will shift demands from the hours with high supply prices to the others to minimize their individual costs.

\section{Conclusion}

In this paper, a distributed optimal day-ahead scheduling for social welfare maximization is proposed, which will be suitable for a future smart grid. In this day-ahead scheduling, the day-ahead market consists of a day-ahead supply market and a day-ahead delivery market. The aggregations of consumers or large consumers purchase generation capacities from the power suppliers in the day-ahead supply market and transmission capacities from the transmission owners in the day-ahead delivery market, respectively. Besides, the hourly supply prices of each power supplier are regulated by the ISO based on his individual hourly demand-supply situations, and the hourly delivery prices of each transmission owner are regulated by the ISO based on his individual hourly demand-delivery situations. This interaction among consumers, power suppliers, and transmission owners will be continued until all the hourly demand-supply and hourly demand-delivery are balanced. Besides, the proposed optimal day-ahead scheduling can be properly matched with a standard ADMM framework, by which a global and fast convergence is guaranteed and the privacy of participants is ensured. The effectiveness of the proposed day-ahead scheduling is verified by the cases studied.

\section{Conflicts of Interest}

The authors declare that there are no conflicts of interest to declare.

\section{Acknowledgments}

This work was partially supported by the National Natural Science Foundation of China under Grants nos. 61673303, 71601188, and 61601334 .

\section{References}

[1] M. Sarker, Y. Dvorkin, and M. Ortega-Vazquez, "Optimal participation of an electric vehicle aggregator in day-ahead energy and reserve markets," IEEE Transactions on Power Systems, vol. 31, no. 5, pp. 3506-3515, 2016.

[2] P. Yang, G. Tang, and A. Nehorai, "A game-theoretic approach for optimal time-of-use electricity pricing," IEEE Transactions on Power Systems, vol. 28, no. 2, pp. 884-892, 2013.

[3] R. Deng, Z. Yang, M. Chow, and J. Chen, "A survey on demand response in smart grids: mathematical models and approaches," IEEE Transactions on Industrial Informatics, vol. 11, no. 3, pp. 570-582, 2015

[4] R. Verschae, T. Kato, and T. Matsuyama, "Energy management in prosumer communities: a coordinated approach," Energies, vol. 9, no. 7, p. 562, 2016.

[5] H. Wu, M. Shahidehpour, A. Alabdulwahab, and A. Abusorrah, "Demand Response Exchange in the Stochastic Day-Ahead Scheduling with Variable Renewable Generation," IEEE Transactions on Sustainable Energy, vol. 6, no. 2, pp. 516-525, 2015.

[6] S. Abedi, G. H. Riahy, S. H. Hosseinian, and A. Alimardani, "Risk-Constrained Unit Commitment of Power System Incorporating PV and Wind Farms," ISRN Renewable Energy, vol. 2011, Article ID 309496, 8 pages, 2011.

[7] G. Chen, C. J. Li, and Z. Y. Dong, "Parallel and Distributed Computation for Dynamical Economic Dispatch," IEEE Transactions on Smart Grid, vol. 8, no. 2, pp. 1026-1027, 2017.

[8] Y. Zhang, N. Rahbari-Asr, J. Duan, and M.-Y. Chow, "Dayahead smart grid cooperative distributed energy scheduling with renewable and storage integration," IEEE Transactions on Sustainable Energy, vol. 7, no. 4, pp. 1739-1748, 2016.

[9] S. Hatami and M. Pedram, "Minimizing the Electricity Bill of Cooperative Users under a Quasi-Dynamic Pricing Model," in Proceedings of the 1st IEEE International Conference on Smart Grid Communications (SmartGridComm), pp. 421-426, Gaithersburg, Md, USA, October 2010.

[10] P. Yang, P. Chavali, E. Gilboa, and A. Nehorai, "Parallel load schedule optimization with renewable distributed generators in smart grids," IEEE Transactions on Smart Grid, vol. 4, no. 3, pp. 1431-1441, 2013.

[11] X. Jin, Y. Mu, H. Jia, J. Wu, X. Xu, and X. Yu, "Optimal day-ahead scheduling of integrated urban energy systems," Applied Energy, vol. 180, pp. 1-13, 2016. 
[12] P. Samadi, H. Mohsenian-Rad, R. Schober, and V. W. S. Wong, "Advanced demand side management for the future smart grid using mechanism design," IEEE Transactions on Smart Grid, vol. 3, no. 3, pp. 1170-1180, 2012.

[13] Y. Zhang and G. B. Giannakis, "Distributed Stochastic Market Clearing with High-Penetration Wind Power," IEEE Transactions on Power Systems, vol. 31, no. 2, pp. 895-906, 2016.

[14] L. Wu, "Impact of price-based demand response on market clearing and locational marginal prices," IET Generation, Transmission \& Distribution, vol. 7, no. 10, pp. 1087-1095, 2013.

[15] C. Zhao, J. Wang, J.-P. Watson, and Y. Guan, "Multi-stage robust unit commitment considering wind and demand response uncertainties," IEEE Transactions on Power Systems, vol. 28, no. 3, pp. 2708-2717, 2013.

[16] R. Deng, G. Xiao, R. Lu, and J. Chen, "Fast distributed demand response with spatially and temporally coupled constraints in smart grid," IEEE Transactions on Industrial Informatics, vol. 11, no. 6, pp. 1597-1606, 2015.

[17] Z. W. Liu, X. Yu, and Z. H. Guan, "Modulated intermittent control in consensus of multi-agent systems," IEEE Transactions on Systems, Man, and Cybernetics: Systems, vol. 47, no. 5, pp. 783793, 2017.

[18] Z.-W. Liu, Z.-H. Guan, X. Shen, and G. Feng, "Consensus of multi-agent networks with aperiodic sampled communication via impulsive algorithms using position-only measurements," IEEE Transactions on Automatic Control, vol. 57, no. 10, pp. 2639-2643, 2012.

[19] H. Li, G. Chen, T. Huang, Z. Dong, W. Zhu, and L. Gao, "Eventtriggered distributed average consensus over directed digital networks with limited communication bandwidth," IEEE Transactions on Cybernetics, vol. 46, no. 12, pp. 3098-3110, 2016.

[20] X. Hu, H. Zhou, Z. Liu, X. Yu, and C. Li, "Hierarchical Distributed Scheme for Demand Estimation and Power Reallocation in a Future Power Grid," IEEE Transactions on Industrial Informatics, vol. 13, no. 5, pp. 2279-2290, 2017.

[21] Y. Wang, L. Wu, and S. Wang, "A Fully-Decentralized Consensus-Based ADMM Approach for DC-OPF With Demand Response," IEEE Transactions on Smart Grid, vol. 8, no. 6, pp. 2637-2647, 2017.

[22] J. N. Tsitsiklis and Y. Xu, "Pricing of fluctuations in electricity markets," European Journal of Operational Research, vol. 246, no. 1, pp. 199-208, 2015.

[23] B. Jiang, A. M. Farid, and K. Youcef-Toumi, "Demand side management in a day-ahead wholesale market: A comparison of industrial \& social welfare approaches," Applied Energy, vol. 156, pp. 642-654, 2015.

[24] D. P. Bertsekas and J. N. Tsitsiklis, Parallel and Distributed Computation: Numerical Methods, vol. 23, Prentice Hall, Englewood Cliffs, NJ, USA, 1989. 


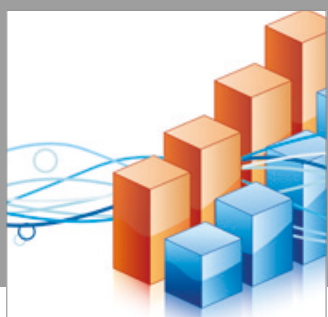

Advances in

Operations Research

vatersals

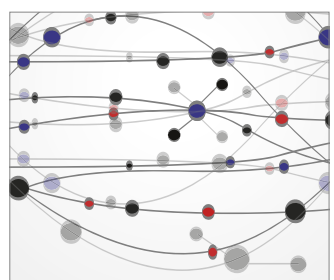

\section{The Scientific} World Journal
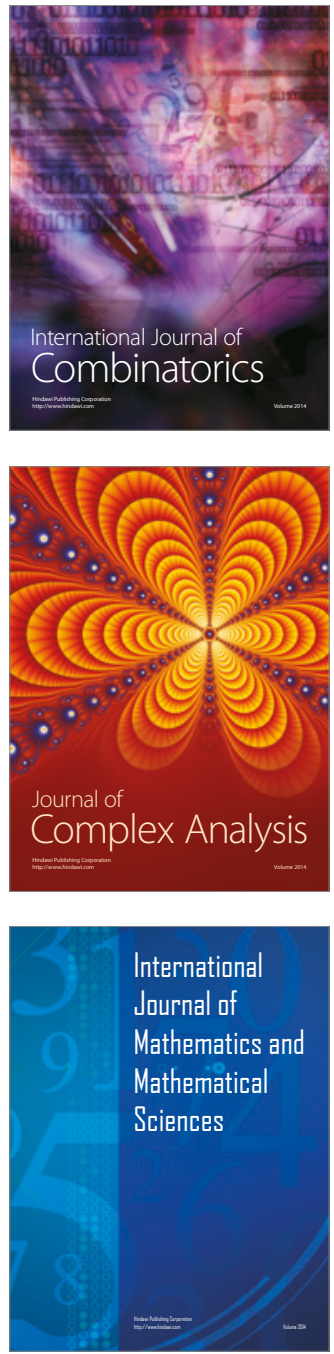
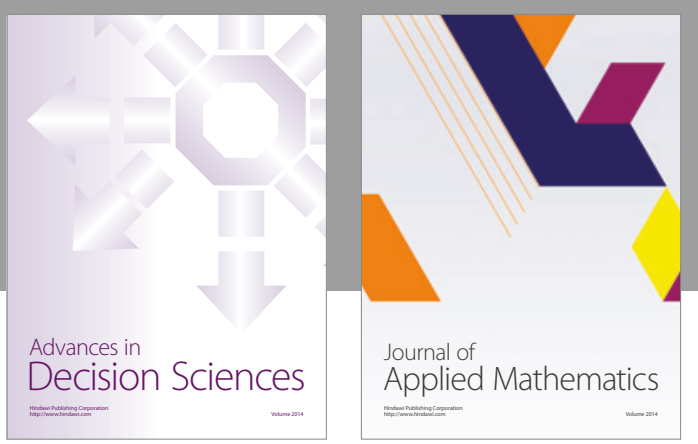

Algebra

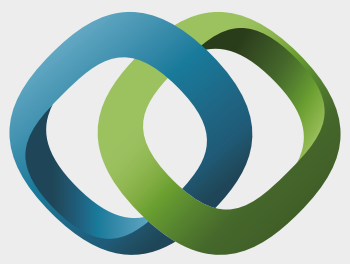

\section{Hindawi}

Submit your manuscripts at

https://www.hindawi.com
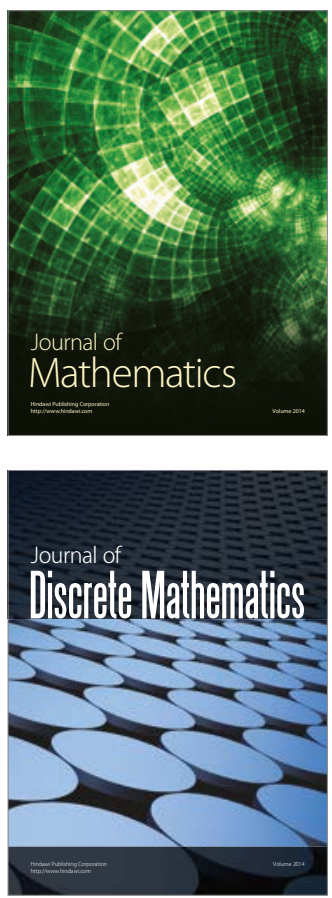

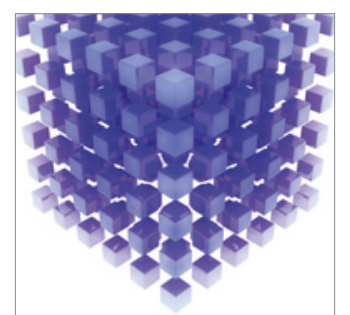

Mathematical Problems in Engineering
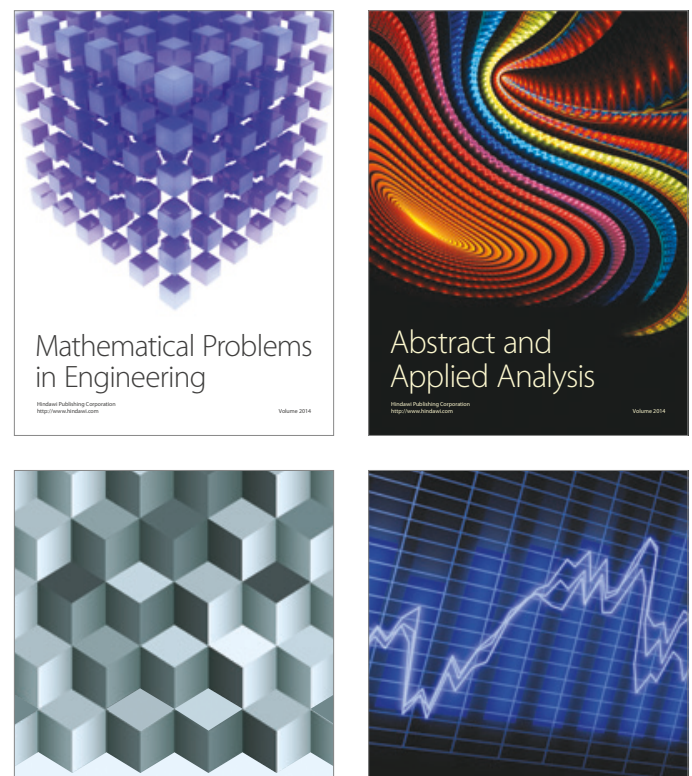

Journal of

Function Spaces

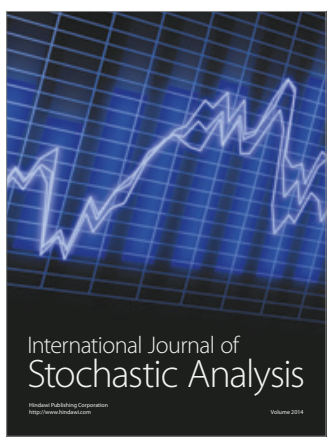

Probability and Statistics
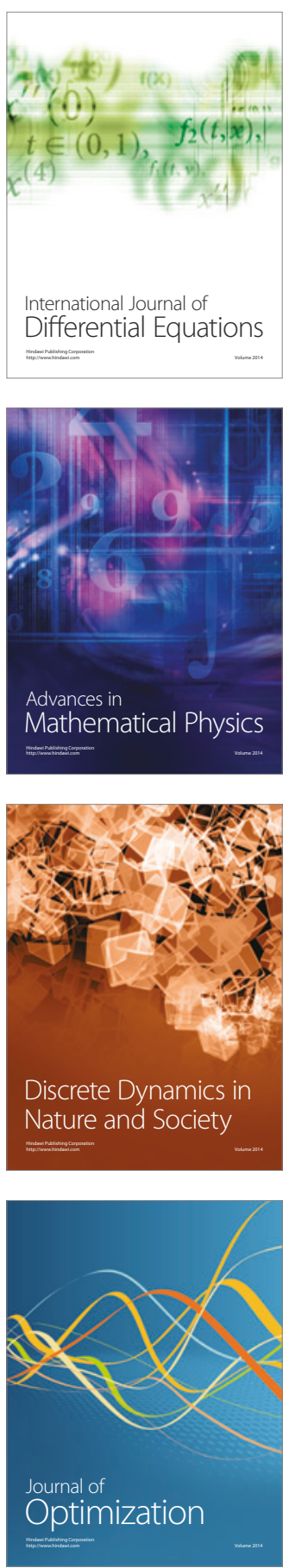